Abstract 71 Table 1 Comparison of NSTEMI patients in 2019 and 2020

\begin{tabular}{|l|c|c|c|}
\hline & $\mathbf{2 0 1 9}$ & $\mathbf{2 0 2 0}$ & $\mathbf{p}-\mathbf{v a l u e}$ \\
\hline Age (years) & $71.2 \pm 12.2$ & $65.0 \pm 12.1$ & $\mathrm{p}<0.01$ \\
\hline NSTEMI patients & 56 & 59 & $\mathrm{P}=\mathrm{NS}$ \\
\hline NSTEMI undergoing angiography & 50 & 53 & $\mathrm{P}=\mathrm{NS}$ \\
\hline $\begin{array}{l}\text { Median Time to angiography } \\
\text { (hours) }\end{array}$ & 68.6 & 14 & $\mathrm{p}<0.01$ \\
\hline Median length of stay (days) & 5 (IQR 2:8) & 2 (IQR 1:4) & $\mathrm{p}<0.01$ \\
\hline Referral to cardiac rehabilitation & $77.4 \%$ & $84.5 \%$ & $\mathrm{P}=\mathrm{NS}$ \\
\hline $\begin{array}{l}\text { Prescription of secondary } \\
\text { prevention medication }\end{array}$ & $69.8 \%$ & $72.4 \%$ & $\mathrm{P}=\mathrm{NS}$ \\
\hline
\end{tabular}

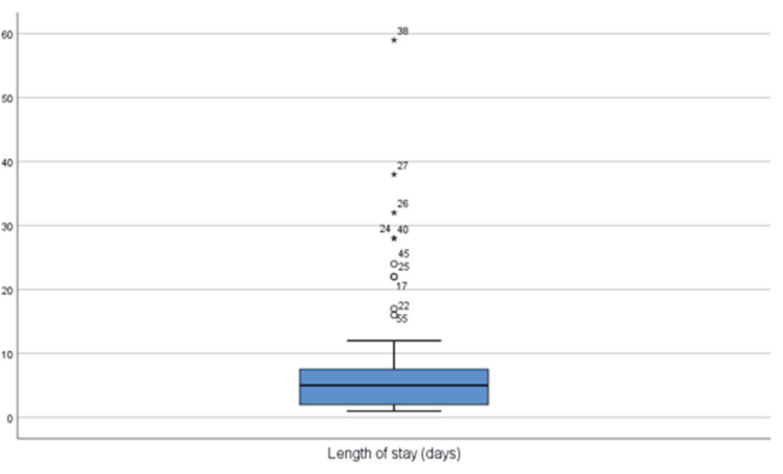

Median for Hospital stay 2020 (2, IQR 1:4)

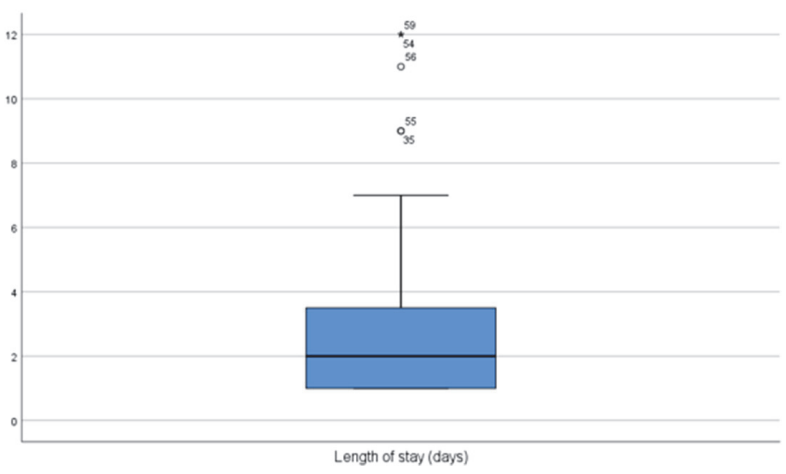

Abstract 71 Figure 1 Box and Whisker Plot for median hospital stay for NSTEMI patients in 2019 and 2020

Median time for Hospital stay 2019 (5, IQR 2:8)

Background The covid-19 pandemic has had a profound impact on healthcare service delivery. Acute cardiovascular care however remains a priority despite the pandemic. Patients presenting with non-ST elevation MI (NSTEMI) have been poorly characterized during the pandemic.

Methods We conducted a retrospective study of patients diagnosed with NSTEMI during the peak of the pandemic between April-May 2020 at our tertiary centre in the UK. Data were collected from electronic patient clinical records including time from admission to angiography, length of stay, mortality, prescription of secondary prevention pharmacotherapy, and referral to cardiac rehabilitation. We compared this data to the same time period in 2019 .

Results As can be seen from table 1, in 2020, the mean age, median time to angiography, and length of stay were all significantly lower than the control period of 2019. Prescription of secondary prevention medication (Aspirin, P2Y12 inhibitor, Beta-blocker, Statin, and ACEi/ARB) and referral to cardiac rehabilitation also improved in 2020, however, neither was statistically significant. During the 2020 period, 1 patient died due to late presentation NSTEMI and multi-organ failure. There were 3 deaths in 2019: complications following coronary bypass surgery, upper GI bleed and a subarachnoid haemorrhage. Table 1 Comparison of NSTEMI patients in 2019 and 202020192020 p-valueAge (years) $71.2 \pm 12.265 .0 \pm 12.1 \mathrm{p}<0.01$ NSTEMI patients $5659 \mathrm{P}=\mathrm{NSNSTEMI}$ undergoing angiography $5053 \mathrm{P}=\mathrm{NS}$ Median Time to angiography (hours) $68.614 \mathrm{p}<0.01$ Median length of stay (days)5 (IQR 2:8)2 (IQR 1:4) p $<0.01$ Referral to cardiac rehabilitation $77.4 \% 84.5 \% \mathrm{P}=\mathrm{NS}$ Prescription of secondary prevention medication $69.8 \% 72.4 \% \mathrm{P}=\mathrm{NS}$.

Conclusion Our results show that the mean age of the patients presenting with NSTEMI during the peak of the Covid-19 pandemic was younger suggesting that elderly patients failed to present. Compared to 2019, there was a significant improvement in patient treatment times with a significantly shorter stay in the hospital, probably as a result of the reduction in elective activity allowing un-restrained access to the catheter labs. We also saw improvement in prescription for secondary prevention and referral to cardiac rehabilitation services during this time suggesting that there may have been improved focus on these aspects of care again.

Conflict of Interest No

\section{ACUTE KIDNEY INJURY FOLLOWING PERCUTANEOUS CORONARY INTERVENTION FOR ACUTE CORONARY SYNDROMES - INCIDENCE, AETIOLOGY, RISK FACTORS AND OUTCOMES}

${ }^{1}$ Elliott Carande, ${ }^{1}$ Karen Brown, ${ }^{1}$ David Jackson, ${ }^{1}$ Nicholas Maskell, ${ }^{2}$ Loukas Kouzaris, ${ }^{3}$ Giles Greene, ${ }^{1}$ Ashraf Mikhail, 1,2Daniel Obaid. 'Swansea Bay University Health Board, Swansea, UK; ${ }^{2}$ Swansea University Medical School; ${ }^{3}$ Public Health Wales

\subsection{6/heartjnl-2021-BCS.72}

Background Acute kidney injury (AKI) is a recognised complication of percutaneous coronary intervention (PCI). We investigated the predictors, aetiology and long-term outcome of AKI following PCI for acute coronary syndromes (ACS).

Methods Two thousand nine-hundred and seventeen patients undergoing PCI for ACS were retrospectively investigated. AKI incidence was identified and cardiovascular and demographic risk factors, and dates of death were collected. 


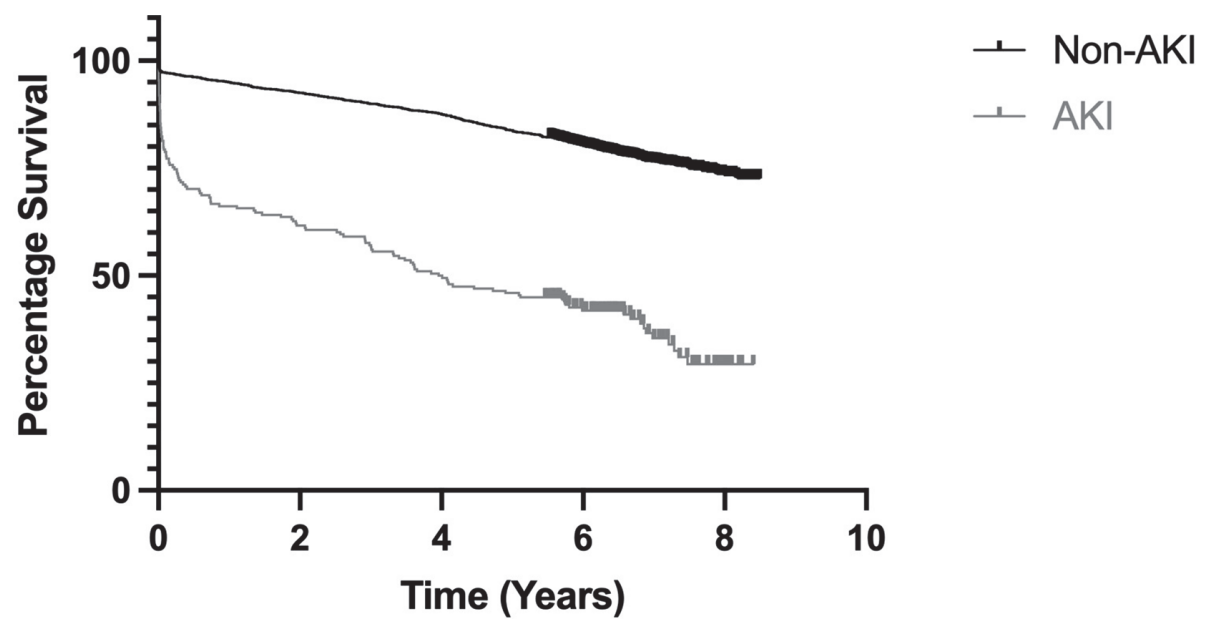

\section{Abstract 72 Figure 1}

Results After exclusion, 198 patients (7.2\% undergoing PCI) had an AKI: $14.1 \%$ of these AKI patients presented in cardiogenic shock, whilst $5.1 \%$ of patients had an elevated ESR and/or eosinophil count and were classified as atheroembolic renal disease (AERD). Statistically significant risk factors for developing AKI were increased age (OR 1.04, 95\% Cl 1.03 to $1.06, \mathrm{p}<0.0001$ ), diabetes (OR $1.56,95 \% \mathrm{Cl} 1.09$ to 2.21 , $\mathrm{p}=0.0129$ ), heart failure (OR $2.30,95 \% \mathrm{Cl} 1.22$ to 4.15 , $\mathrm{p}=0.0073$ ), femoral access (OR 1.47, 95\% Cl 1.02 to 2.10, $\mathrm{p}=0.0357$ ) and cardiogenic shock on arrival (OR 2.92, 95\% $\mathrm{Cl} 1.72$ to $4.81, \mathrm{p}<0.0001)$. Significant association with mortality at 1-year was found in patients with an AKI (OR 4.33, $95 \% \mathrm{Cl} 2.89$ to $6.43, \mathrm{p}<0.0001$ ), age (OR $1.08,95 \% \mathrm{Cl}$ 1.06 to $1.09, \mathrm{p}<0.0001$ ), heart failure (OR $1.92,95 \% \mathrm{Cl}$ 1.05 to $3.44, \mathrm{p}=0.032$ ), femoral access (OR 2.05, 95\% Cl 1.41 to $2.95, \mathrm{p}=0.0001$ ), and cardiogenic shock (OR 3.63, $95 \% \mathrm{Cl} 2.26$ to $5.77, \mathrm{p}<0.0001)$. Analysis of survival demonstrated a hazard ratio of mortality of 4.23 in the AKI group when compared to non-AKI patients $(95 \% \mathrm{Cl} 3.00$ to 5.98 , $\mathrm{p}<0.0001$ ) (see figure 1). Significant associations with 1-year mortality in AKI patients were age (OR 1.04, 95\% $\mathrm{Cl} 1.01$ to 1.07, $\mathrm{p}=0.011$ ), and cardiogenic shock (OR 4.40, 95\% Cl 1.56 to $10.90, \mathrm{p}=0.004)$. Patients with AERD AKI had a 1year mortality rate of $40.0 \%$ and a 1 -year renal replacement therapy requirement of $22.2 \%$. This was compared to a 1 year mortality rate of $33.8 \%$ in AKI patients of any aetiology, and a renal replacement therapy requirement of $8.0 \%$.

Conclusion AKI after urgent PCI is strongly associated with worse outcome. Risk factors for developing AKI were age, diabetes, heart failure, femoral access and cardiogenic shock. Risk of mortality at 1-year were the development of AKI, age, femoral access and cardiogenic shock. AERD occurred in $5.1 \%$ of those who develop an AKI and is an often-overlooked condition with poor outcome and likelihood of longterm renal replacement requirement. Early identification of patients is important to provide appropriate supportive care.

Conflict of Interest None

\section{IMMERSIVE CLINICAL SIMULATION FOR ASSESSMENT OF ULTRASOUND-GUIDED PERICARDIOCENTESIS PROCEDURES FOR TRAINEES}

Kamatamu Amanda Mbonye, Helen Mills, Khalid Barakat. Barts Health NHS Trust, London, UK
Introduction It is well documented that part-task trainers are highly effective in practical skills training, including pericardiocentesis. ${ }^{1}{ }^{2}$ However, the use of immersive simulation to create high-fidelity clinical scenarios for summative assessment of pericardiocentesis is a novel approach. Cardiology trainees in the UK are expected to demonstrate Level 3 competency (perform the procedure unsupervised and deal with complications) in pericardiocentesis in order to meet the curriculum requirements to achieve their Certificate of Completion of Training (CCT). Given the high risk of complications associated with pericardiocentesis, it is now increasingly performed by interventional cardiologists in specialist centres, resulting in fewer opportunities for trainees to observe and practice this procedure during their training. This has become more apparent during the COVID-19 pandemic when normal clinical activities were largely put on hold, resulting in an increase in demand for simulation-based training and assessment of practical procedures.

Methods Four cardiology trainees attended a series of two teaching sessions held 1 month apart which involved an immersive simulated scenario on the management of a patient with life-threatening cardiac tamponade. This included a period of teaching from a qualified trainer, followed by an observed assessment whereby trainees attended to a simulated patient and initiated relevant treatment, including successfully performing pericardiocentesis whilst interacting with colleagues and the patient. The procedure was performed using an 'Ultrasound Guided Pericardiocentesis Simulator' and a patient actor. Trainees were assessed using the Joint Royal Colleges Physician Training Board (JRCPTB) Directly Observed Procedures (DOPS) proforma for pericardiocentesis. The assessment comprised of 9 different domains graded (A-F), and a point score (1-6) was assigned according to their level of competency, generating an overall assessment score out of 54 points and mean score out of 6 points.

Results Three out of the 4 trainees improved their mean assessment scores by an average of $22 \%$ in subsequent sessions (figure 1). Trainee 1 was already fully competent on initial assessment (initial mean score 6/6), demonstrated sustained competency throughout subsequent assessments and consequently fulfilled their training requirements for pericardiocentesis.

Conclusion Immersive simulation is an effective, low-risk and high-fidelity method of training and assessment of pericardiocentesis for cardiology trainees. Although additional trainee 